\title{
Triatominae et CActaceae : UN RISQUE POUR LA TRANSMISSION DE LA TRYPANOSOMOSE AMÉRICAINE DANS LE PÉRIDOMICILE (NORD-EST DU BRÉSIL)
}

\author{
EMPERAIRE L. * \& ROMAÑA C.A.**
}

Summary: TRIATOMINAE AND CACTACEAE: A RISK FOR THE TRANSMISSION OF THE AMERICAN TRYPANOSOMIASIS IN THE PERIDOMICILARY SPACE (NORTHEAST BRAZIL)

Field observations carried in semi-arid Brazil Northeast point out the frequent association, in the peridomiciliary space, between a cactus, Cereus jamacaru, the occurrence of nests in its branches and the occurrence of two species of insects vectors of Trypanosoma cruzi, pathogenic agent of Chagas disease: Rhodnius neglectus and Triatoma pseudomaculata. The analysis of the architectural variables of this Cactaceae shows that the presence of nests, and thus of insects, depends on the traditional practices of management of this cactus. This study underlines the relevance of an integrated approach of the ecology of

Triatominae for the identification of factors of risk.

KEY WORDS : risk, American trypanosomiasis, Northeast Brazil, environment management, Cactaceae, "caatinga", Rhodnius neglectus, Triatoma pseudomaculata.

\section{Résumé :}

Les observations de terrain réalisées dans le nord-est du Brésil ont montré la fréquente association, dans l'espace péridomiciliaire, entre un cactus, le Cereus jamacaru, la présence de nids dans ses branches et celle de Rhodnius neglectus et de Triatoma pseudomaculata, espèces vectrices du parasite Trypanosoma cruzi, agent de la maladie de Chagas. L'analyse des variables architecturales de cette Cactaceae montre que la présence de nids, et donc d'insectes, est inféodée aux pratiques traditionnelles de gestion de ce cactus. Cette étude souligne l'intérêt d'une approche intégrée de l'écologie des Triatominae pour l'identification des variables indicatrices de risque.

MOTS CLÉS : risque, trypanosomose américaine, nord-est du Brésil, gestion de l'environnement, Cactaceae, "caatinga", Rhodnius neglectus, Triatoma pseudomaculata.

\section{$\mathrm{I}$} es Cactaceae du milieu sylvestre sont considérées dans la littérature comme un habitat potentiel de Triatominae également sylvestres. Carcavallo et al. (1998) en citent plusieurs exemples. Marroquín et al. (2000a et b) relèvent l'association entre Triatoma ryckmani Zeledón \& Ponce, 1972 et deux Cactaceae, Stenocereus sp. et Machaerocereus gummosus (Engelm.) Britton \& Rose dans les formations semi-arides d'Amérique Centrale. De même, Vezzani et al. (2001) ont capturé T. guasayana Wygodzinsky \& Abalos dans Opuntia quimilo Schum. dans les forêts sèches du nord de l'Argentine.

Nos observations de terrain réalisées dans le nord-est brésilien, autre zone semi-aride néotropicale, indiquent que l'association de Cactaceae et de Triatominae ne se limite pas au milieu sylvestre, mais qu'elle se rencontre aussi dans l'environnement péridomiciliaire. En effet, nous avons relevé la présence de T. pseudomaculata Corrêa \& Espínola, 1964 et de Rhodnius neglectus Lent, 1954, soit deux espèces vectrices de la trypanosomose américaine, dans une Cactaceae, le "mandacaru" ou

\footnotetext{
* Institut de Recherche pour le Développement, UR 016 - Caractérisation et contrôle des populations de vecteurs - LIN/IRD, 911, avenue Agropolis, BP 64501, 34394 Montpellier Cedex 5, France.

** Université de Paris 5, Faculté de Médecine.

Correspondance : Laure Emperaire. E-mail : emperair@uol.com.br
}

Cereus jamacaru DC., fréquemment plantée à proximité des habitations.

Contrairement aux cas précédents où les insectes avaient été relevés dans des tiges sèches, les Triatominae se trouvaient ici dans des nids d'oiseaux ou de rongeurs et non directement associés à la plante. La présence de Triatominae dans des nids d'oiseaux anthropophiles et de rongeurs a déjà été soulignée par Carcavallo \& Martínez (1985) qui indiquent l'occurrence de Psammolestes sp. dans des nids de Phacellodomus rufifrons (Wied-Neuwied, 1821), Furnariidae. Sick (1984) mentionne P. arthuri Pinto, 1926 dans des nids de ce Furnariidae au Venezuela. En Argentine, Triatoma delpontei Romaña \& Abalos, 1947 et T. platensis Neiva, 1913 ont été relevés dans des nids d'un Psittacidae, Myiopsitta monachus (Boddaert, 1783) et de deux Furnariidae, Phacellodomus sp. et Pseudoseisura lophotes (Reichenbach, 1853), soit dans des espèces anthropophiles (Carcavallo \& Martínez, 1985).

En raison du risque constitué par la présence d'insectes vecteurs à proximité des habitations, il importait de s'interroger sur les déterminants de l'association entre Cactaceae, espèces anthropophiles et Triatominae. Il s'agissait en particulier de comprendre les pratiques de gestion à l'origine de la présence de cette Cactaceae dans le péridomicile. En effet, afin de définir des stra- 
tégies efficaces de contrôle des insectes vecteurs de Trypanosoma cruzi Chagas 1909, agent pathogène de la trypanosomose américaine, il est nécessaire d'avoir une meilleure connaissance non seulement de leur écologie et de leur dispersion, mais aussi des pratiques humaines susceptibles de favoriser leur présence dans un environnement donné.

\section{MATÉRIEL ET MÉTHODES}

\section{LA ZONE D'ÉTUDE}

I a région de Curaçá, dans le nord de l'état de Bahia (figure 1), est située dans le grand biome des formations xérophytiques à saisonnalité marquée, les "caatingas", dont les faciès vont de la forêt sèche à des formations steppiques. Selon la classification de Köppen, le climat local est de type semi-aride (BShw). Les précipitations annuelles sont d'environ $300 \mathrm{~mm}$ avec une grande variabilité interannuelle. La saison sèche, bien marquée par l'aspect décidu de la végétation, s'étend de mai à octobre. La température moyenne annuelle est de $27^{\circ} \mathrm{C}$ et l'humidité relative varie entre 48 et $56 \%$ (DCA, UFPb).
La densité moyenne de la population rurale dans cette commune est de 2,8 habitants $/ \mathrm{km}^{2}$ (Brasil, IBGE, 2000) avec un habitat en hameaux dispersés. Les contraintes climatiques modèlent très fortement les activités humaines qui sont centrées sur l'élevage extensif de bovins, caprins ou ovins, et une petite agriculture de subsistance fondée sur le maïs, les haricots et le manioc. L'élevage, moteur d'une colonisation qui a avancé du littoral vers l'intérieur des terres dès le XVII ème siècle (Lopes, 2000), est à l'origine d'une forte dégradation des paysages. Les formations végétales, en général ouvertes, présentent une faible diversité floristique de ligneux et une phytomasse réduite.

Pour pallier la pénurie d'aliments pour le bétail durant la saison sèche, les éleveurs plantent fréquemment à proximité des maisons des "mandacarus". Durant les périodes de soudure, les branches (dénommées articles dans le cas des Cactaceae), aux tissus mucilagineux riches en eau, sont coupées et données aux animaux une fois leurs longues épines brûlées. Ces cactus réitèrent abondamment et, coupés plusieurs années de suite, ils présentent un port touffu particulier alors que les individus non coupés ont un port élancé.

Le "mandacaru" est un cactus de type columnaire qui atteint 7 à 8 mètres de hauteur. Sa durée de vie avoisine

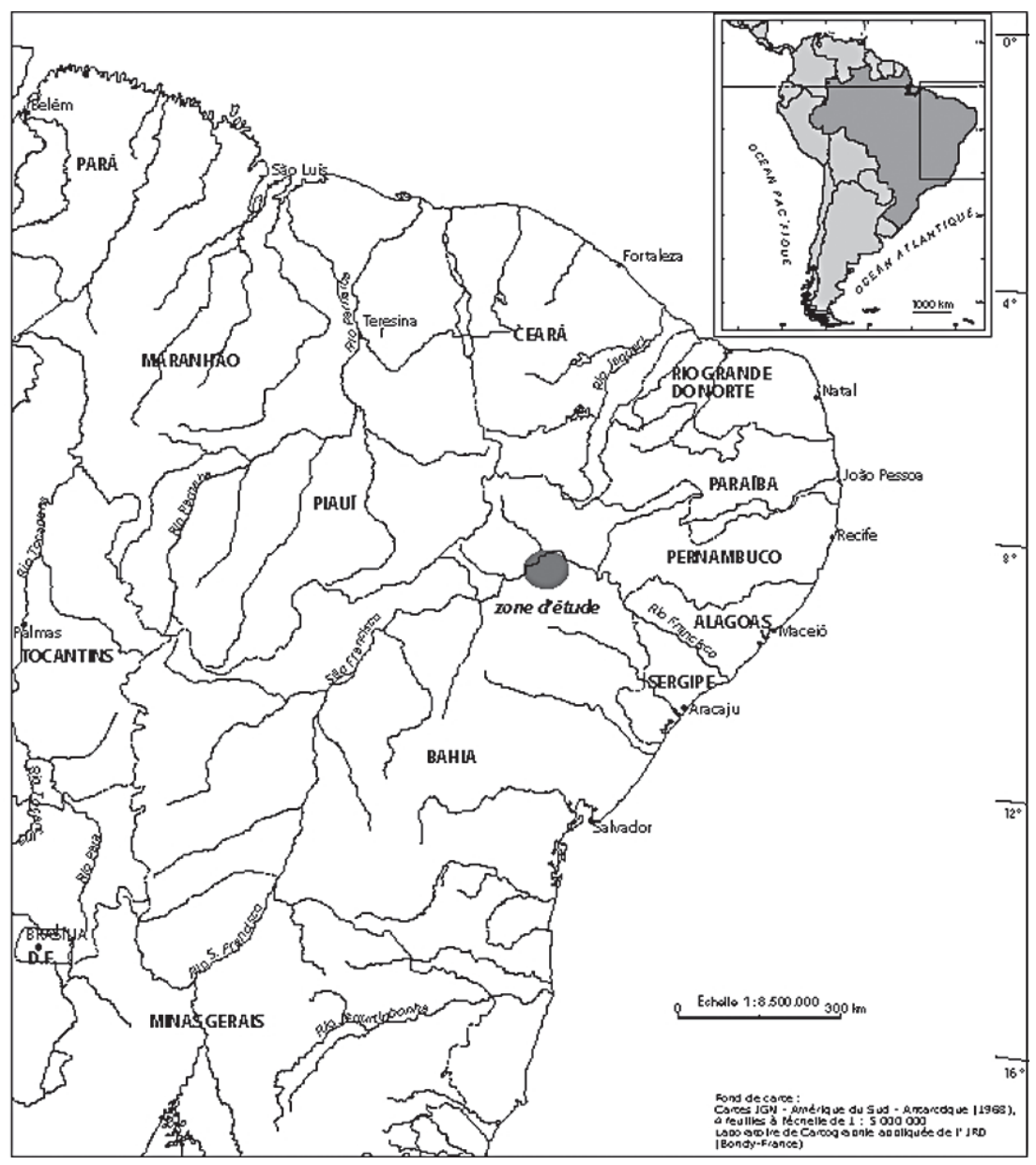

Fig. 1. - Situation géographique de la zone d'étude. 


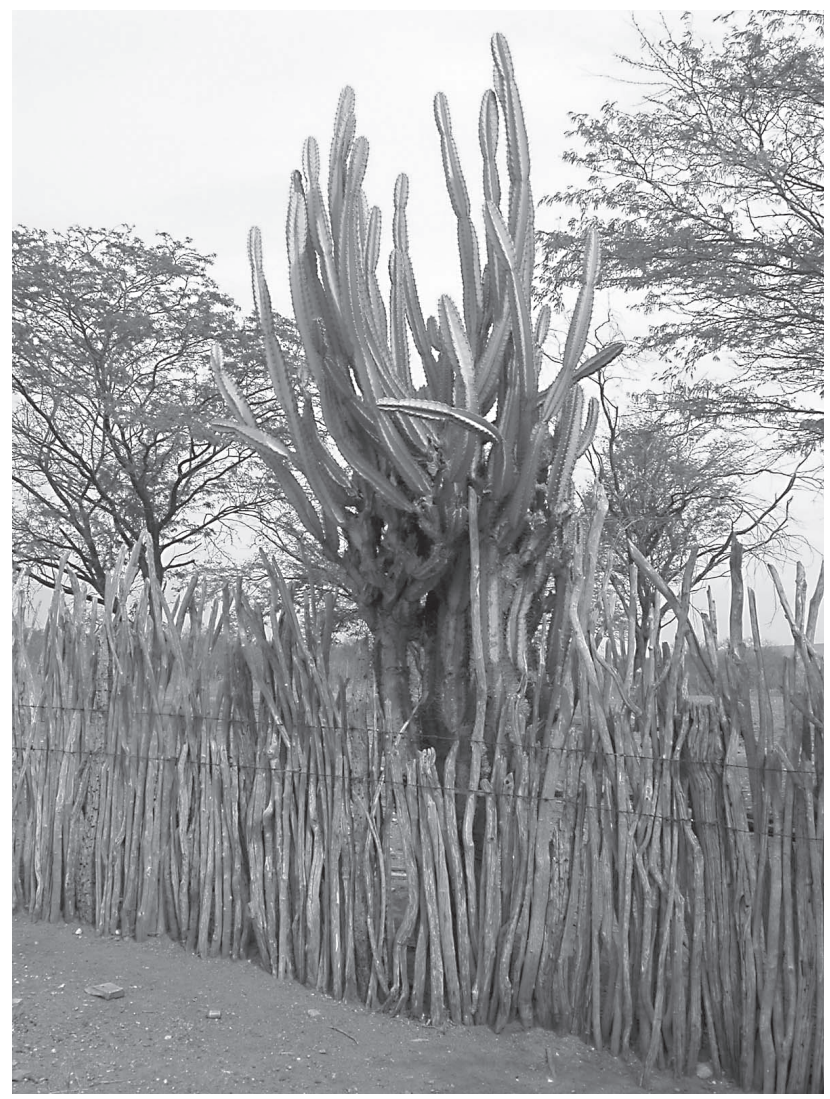

Fig. 2. - "Mandacaru" intercalé dans une palissade.

le siècle. Il est planté isolément ou intercalé dans des palissades ou clôtures (figure 2). Fréquent à l'état spontané ou sub-spontané dans toute la zone sèche du nord-est, il n'est cependant mis en culture que dans les zones les plus arides comme celle de Curaçá. Outre son rôle fourrager, il présente quelques utilisations secondaires, mais surtout, il est l'espèce emblématique du nord-est du Brésil où, en ville, il orne fréquemment places publiques et jardins.

\section{LES SITES D'ÉTUDE}

L'étude a été menée dans la partie nord de la commune de Curaçá, entre $9^{\circ} 01^{\prime}$ S et $9^{\circ} 05^{\prime} \mathrm{S} / 39^{\circ} 47^{\prime} \mathrm{W}$ et $39^{\circ} 51^{\prime} \mathrm{W}$, à une altitude comprise entre 300 et $400 \mathrm{~m}$. Le paysage est celui d'une suite de plateaux faiblement entaillés par des cours d'eau temporaires et parsemés d'inselbergs calcaires. Les zones d'habitation se situent dans des formations ouvertes, dégradées, avec quelques arbustes ou arbrisseaux isolés.

Les relevés ont eu lieu en août 2002 et en avril 2003, soit respectivement en milieu de saison sèche et en fin de saison des pluies. Ils ont été réalisés dans trois sites caractérisés par la présence de Cereus jamacaru dans du péridomicile. Pour les sites $n^{\circ} 1051$ et $n^{\circ} 1052$, il s'agit d'un péridomicile abandonné depuis une trentaine d'années ; ils couvrent respectivement 2 et 5,5 ha.
Le site $\mathrm{n}^{\circ} 1051$ est hétérogène, avec du péridomicile abandonné - reconnaissable à des amoncellements de terre, des restes de palissades de bois plus ou moins décomposées -, quelques petits bosquets de "caatinga" de quelques dizaines de mètres carrés et une zone de "caatinga" arbustive ouverte. Le site n 1052 ne comprend que du péridomicile abandonné avec les mêmes éléments que dans le $n^{\circ} 1051$. Le site $n^{\circ} 1059$ s'étend sur 45 ha et recouvre le péridomicile de six maisons dont une seule est abandonnée. Les espaces propres à chaque maisonnée y sont marqués par un important réseau de palissades avec des "mandacarus" intercalés. Ces clôtures délimitent tant les espaces de chaque maisonnée (jardins, poulaillers, potagers,...) que ceux réservés à l'élevage (lieu de traite, de tri des bêtes,...). Ce péridomicile ne comprend que quelques arbres isolés plantés à des fins fourragères, médicinales ou d'ombrage et de nombreux "mandacarus"; la végétation environnante est arbustive ouverte, très dégradée.

\section{RELEVÉS DE TERRAIN}

La position des "mandacarus" de plus de $2 \mathrm{~m}$ de hauteur (soit potentiellement porteurs de nids) a été relevée au GPS et reportée sur le plan de chaque site. Les principaux éléments structurant le paysage ont été cartographiés (chemins, réseau hydrique, formations végétales, arbres, habitations, vestiges d'habitation et clôtures). Chaque "mandacaru" a été photographié, ses paramètres architecturaux relevés (hauteur totale, diamètre à 1,3 m, hauteur de lignification, nombre total de branches) et la végétation environnante (péridomicile, "caatinga" arborée ou arbustive ouverte) décrite. Les nids ont été repérés, identifiés à partir d'informations locales, et disséqués. Les Triatominae capturés ont été déterminés au Laboratoire de parasitologie de l'Université de Brasília selon Lent \& Wygodzinsky (1979), les stades de développement caractérisés et le taux d'infection par T. cruzi quantifié.

\section{TRAITEMENT DES DONNÉES}

Les tests de Mann-Whitney et de Kruskal-Wallis, non paramétriques, ont été appliqués à l'étude des corrélations entre variables architecturales, présence/absence de nids et présence/absence de Triatominae. Le test du Khi-2 a été utilisé pour le traitement des variables qualitatives, relations environnement/présence de nids/ présence d'insectes. Le seuil de confiance retenu est de $5 \%$. Le logiciel utilisé est Statview ${ }^{\circledR}$.

\section{RÉSULTATS}

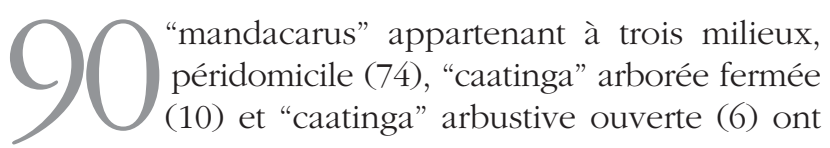


été relevés. 35 étaient porteurs de nids (39\%); parmi ces derniers, trois n'ont pas été disséqués car habités et 11 étaient positifs pour la présence de Triatominae dans les nids (soit $31 \%$ des cactus porteurs de nids). Les deux espèces d'insectes identifiées ont été $T$. pseudomaculata et $R$. neglectus, présents isolément dans respectivement quatre et deux cactus, et ensemble dans cinq cactus (tableau I) sans que l'association entre les deux espèces soit significative.

Selon les informateurs locaux, les nids ont été construits, ou sont occupés, par des "sabiás" (Turdidae), "juritis" et "pombinhas" (Columbidae), "casacos-de-couro" (Furnariidae, Pseudoseisura cristata (Spix, 1824)), "garrinchas" (Troglodytidae), "almas-de-gato" (Cuculidae, Piaya cayana (Linnaeus, 1766)) et de petits rongeurs non identifiés. Un total de 107 insectes ont été capturés dans 14 nids localisés sur les 11 "mandacarus" étudiés (5,3 $\pm 6,1$ insectes en moyenne); 72 insectes appartenaient à l'espèce $R$. neglectus (moyenne $=9,0 \pm 8,3$ insectes/cactus) et 35 à $T$. pseudomaculata (2, $4 \pm 2,9$ insectes/cactus).

On n'observe pas de différence significative dans la présence de nids ou de Triatominae entre les différents environnements végétaux ou entre relevés. L’absence de nids dans la 'caatinga' arbustive ouverte n'est pas statistiquement significative. En revanche, l'analyse des variables architecturales montre que le port des $C$. jamacaru varie de manière significative selon le type de milieu. Il existe un ensemble de cactus petits et grêles dans la "caatinga" arbustive ouverte et un autre de cactus de diamètres et hauteurs plus importants, dans la "caatinga" arborée et le péridomicile. Le nombre total d'articles croit de l'ensemble 'caatinga' arbustiveherbacée (moyenne 6,2), à "caatinga" arborée $(17,8)$, à péridomicile $(21,7)$.

Les valeurs élevées des variables architecturales des Cereus jamacaru sont positivement associées à la présence de nids, soit d'écotopes susceptibles d'héberger des Triatominae. Les variables diamètre et nombre d'articles sont hautement significatives de cette relation (tableau IIA). Parmi les cactus porteurs de nids, on relève une corrélation positive entre la présence d'insectes et le nombre d'articles de Cereus (tableau IIB). R. neglectus est principalement inféodé aux cactus très ramifiés $(\mathrm{p}=0,0221$, $\mathrm{U}=42,5$ ) alors que $T$. pseudomaculata est indifférent aux variables analysées $(\mathrm{p}>0,05)$.

Le fait que le péridomicile soit en activité ou abandonné n'induit de différence significative ni dans la distribution des nids ni dans celle des insectes, que cela soit pour $R$. neglectus ou T. pseudomaculata ce qui tend à montrer que c'est le port même du cactus, et non l'environnement créé par les activités quotidiennes de l'homme, qui est déterminant dans l'installation des insectes.

L'échantillon est trop restreint pour mener une analyse statistique plus fine, néanmoins une relation entre les pratiques de gestion de ce cactus menant à un port touffu et la présence d'insectes vecteurs apparaît nettement.

Quant aux populations d'insectes présentes, on observe deux distributions différentes selon l'espèce considérée

\begin{tabular}{|c|c|c|c|c|}
\hline & Péridomicile & $\begin{array}{c}\text { "Caatinga" } \\
\text { arborée fermée }\end{array}$ & $\begin{array}{c}\text { "Caatinga" } \\
\text { arbustive ouverte }\end{array}$ & Total \\
\hline Cereus jamacaru relevés & 74 & 10 & 6 & 90 \\
\hline Cereus jamacaru positifs pour des nids (\%) & $30(40,5)$ & $4(40,0)$ & $1(16,7)$ & $35(38,9)$ \\
\hline Cereus jamacaru positifs pour les Triatominae (\%) & $10(33,3)$ & $1(25,0)$ & $0(0)$ & $11(31,4)$ \\
\hline pour T. pseudomaculata (\%) & $8(26,7)$ & $1(25,0)$ & $0(0)$ & $9(25,7)$ \\
\hline pour $R$. neglectus (\%) & $7(23,3)$ & $0(0)$ & $0(0)$ & $7(20,0)$ \\
\hline pour $T$. pseudomaculata et $R$. neglectus (\%) & $5(16,6)$ & $0(0)$ & $0(0)$ & $5(14,3)$ \\
\hline
\end{tabular}

Tableau I. - Nombre de Cereus jamacaru positifs pour la présence de nids et positifs pour T. pseudomaculata et $R$. neglectus selon les différentes formations végétales relevées.

\begin{tabular}{|c|c|c|c|c|c|c|c|}
\hline & & & $\begin{array}{c}\text { Nombre } \\
\text { d'individus }\end{array}$ & $\begin{array}{c}\text { Hauteur } \\
\text { totale }\end{array}$ & $\begin{array}{c}\text { Hauteur de } \\
\text { lignification }\end{array}$ & Diamètre & $\begin{array}{c}\text { Nombre } \\
\text { d'articles }\end{array}$ \\
\hline \multirow[t]{4}{*}{ A } & Ensemble des Cereus jamacaru des relevés & Présence de nids & 35 & $4,10 \mathrm{~m}$ & $1,70 \mathrm{~m}$ & $19,0 \mathrm{~cm}$ & 25,0 \\
\hline & & Absence de nids & 55 & $3,50 \mathrm{~m}$ & $1,40 \mathrm{~m}$ & $15,0 \mathrm{~cm}$ & 9,0 \\
\hline & & $\mathrm{U}$ & & 669,0 & 572,5 & 529,0 & 349,5 \\
\hline & & $\mathrm{p}$ & & 0,0151 & 0,0012 & 0,0003 & $<0,0001$ \\
\hline \multirow[t]{4}{*}{ B } & Cereus jamacaru positifs pour les nids & Présence d'insectes & 11 & $4,3 \mathrm{~m}$ & $2,0 \mathrm{~m}$ & $22,0 \mathrm{~cm}$ & 32,0 \\
\hline & & Absence d'insectes & 24 & $4,1 \mathrm{~m}$ & $1,7 \mathrm{~m}$ & $18,5 \mathrm{~cm}$ & 24,0 \\
\hline & & $\mathrm{U}$ & & 118,5 & 111,5 & 102,0 & 74,0 \\
\hline & & $\mathrm{p}$ & & 0,6314 & 0,4663 & 0,2864 & 0,0393 \\
\hline
\end{tabular}

Tableau II. - Valeurs médianes des variables architecturales des Cereus jamacaru relevées selon la présence/absence de nids ; valeurs de U et de $\mathrm{p}$ du test de Mann-Whitney (en grisé : $\mathrm{p}<0,05$ ). 


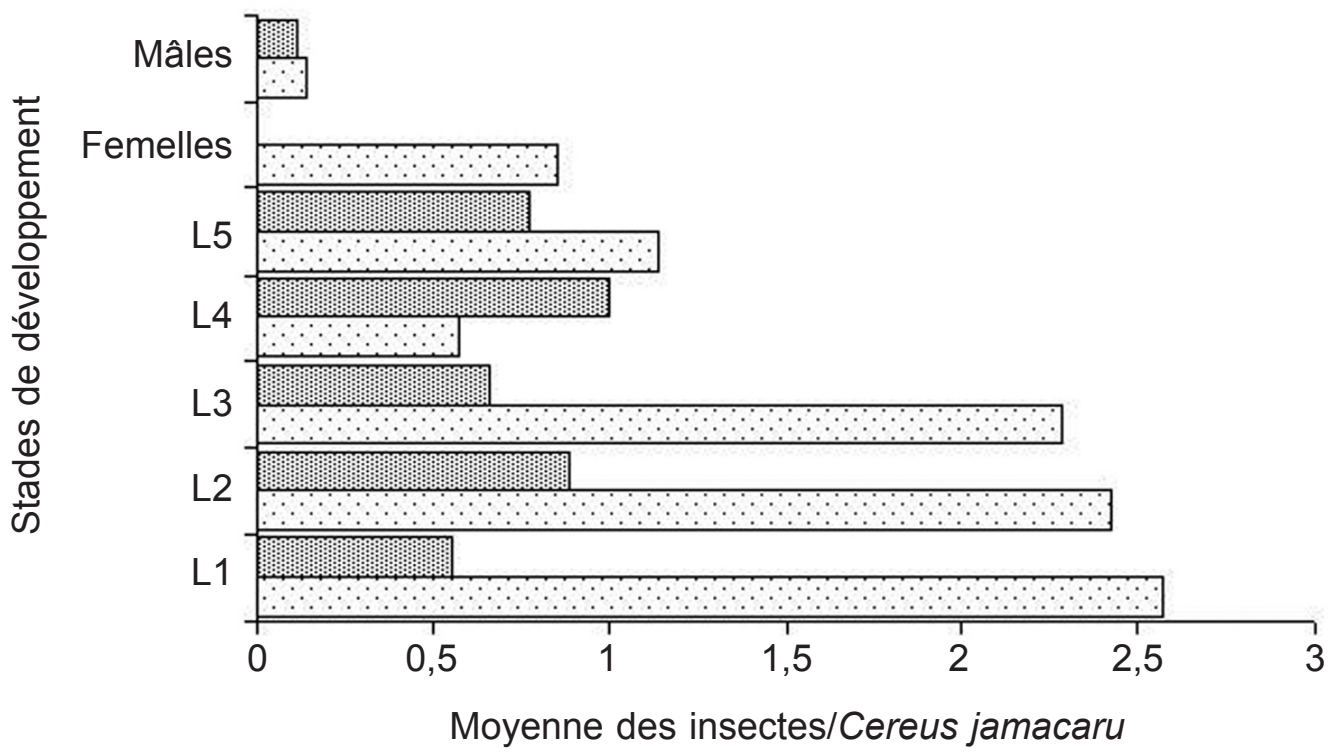

Fig. 3. - Pyramide des stades de développement de Rhodnius neglectus et de Triatoma pseudomaculata capturés dans les Cereus jamacaru.

Rhodnius neglectus 橉 Triatoma pseudomaculata

(figure 3). Pour R. neglectus, l'abondance des premiers stades était importante ce qui générait une pyramide avec une ample base, le 4ème stade étant le moins représenté. Au contraire, chez T. pseudomaculata, la structure des classes d'âge ne montrait aucune tendance. Quelle que soit la saison, le nombre d'insectes de $R$. neglectus (saison humide : 9,0 insectes/"mandacaru"; saison sèche : 11,3) restait bien supérieur à celui de $T$. pseudomaculata (saison humide : 3,0; saison sèche : 4,0). De plus, la reproduction de $R$. neglectus semblait s'effectuer pendant la saison sèche car des œufs, des larves de premier stade et des femelles en nombre conséquent ont été trouvés lors des dissections des nids. Des reptiles et/ou des œufs d'oiseaux étaient présents dans huit des 14 (57\%) nids infestés par les insectes, dans six des huit nids à $R$. neglectus et quatre des 11 nids à T. pseudomaculata.

Aucun des insectes examinés (45,7\% et 54,3\% de la population totale de $R$. neglectus et de T. pseudomaculata respectivement) n'était parasité par T. cruzi.

\section{DISCUSSION}

L a distribution des deux espèces capturées, $R$. neglectus et $T$. pseudomaculata, s'étend au nord-est et au centre du Brésil, dans des formations à dominance xérophytique. Les deux espèces sont caractérisées par différents auteurs comme étant ornithophiles. Ainsi, la première a été souvent trouvée dans les nids situés dans différents palmiers tandis que la seconde a été capturée non seulement en milieu sylvestre mais aussi dans les poulaillers du péridomicile (Alencar,
1980). Cependant, l'analyse des stades de population montre que des deux espèces, $R$. neglectus semble être la mieux adaptée aux ressources trophiques disponibles dans les nids des "mandacarus", reptiles et oiseaux. Cette relation expliquerait l'absence de T. cruzi dans les intestins des insectes examinés car, reptiles et oiseaux, ne peuvent, pour des raisons physiologiques, constituer des réservoirs du parasite.

Les résultats montrent d'autre part que le Cereus jamacaru constitue dans l'environnement péridomiciliaire un écotope favorable à des insectes potentiellement vecteurs. La présence de nids et de Triatominae est liée à l'architecture du "mandacaru" qui elle-même résulte d'un certain type de gestion de cette ressource. La présence de $R$. neglectus est étroitement associée à celle de nids dans des cactus au port touffu. T. pseudomaculata est davantage lié à la seule présence de nids, quel que soit le port du cactus. Une hypothèse serait que le "mandacaru” est pour l'espèce $R$. neglectus un écotope primaire sylvestre et péridomiciliaire (présence dans les nids de colonies entières, y compris d'adultes femelles) alors que pour les populations de T. pseudomaculata, celui-ci ne serait qu'un relais lors de sa dispersion.

Les relations entre gestion du "mandacaru" par les agriculteurs éleveurs de la région de Curaçá, présence de nids et d'insectes vecteurs sont représentées dans la figure 4. Ce schéma met en jeu quatre ensembles de facteurs qui relèvent de contraintes régionales, de la gestion du végétal, de la présence d'animaux anthropophiles et de celle d'insectes vecteurs avec l'enchâ̂nement suivant : 1) la principale activité de la région, marquée par une saison sèche intense, est l'élevage extensif; 2) la bonne gestion du troupeau implique un 


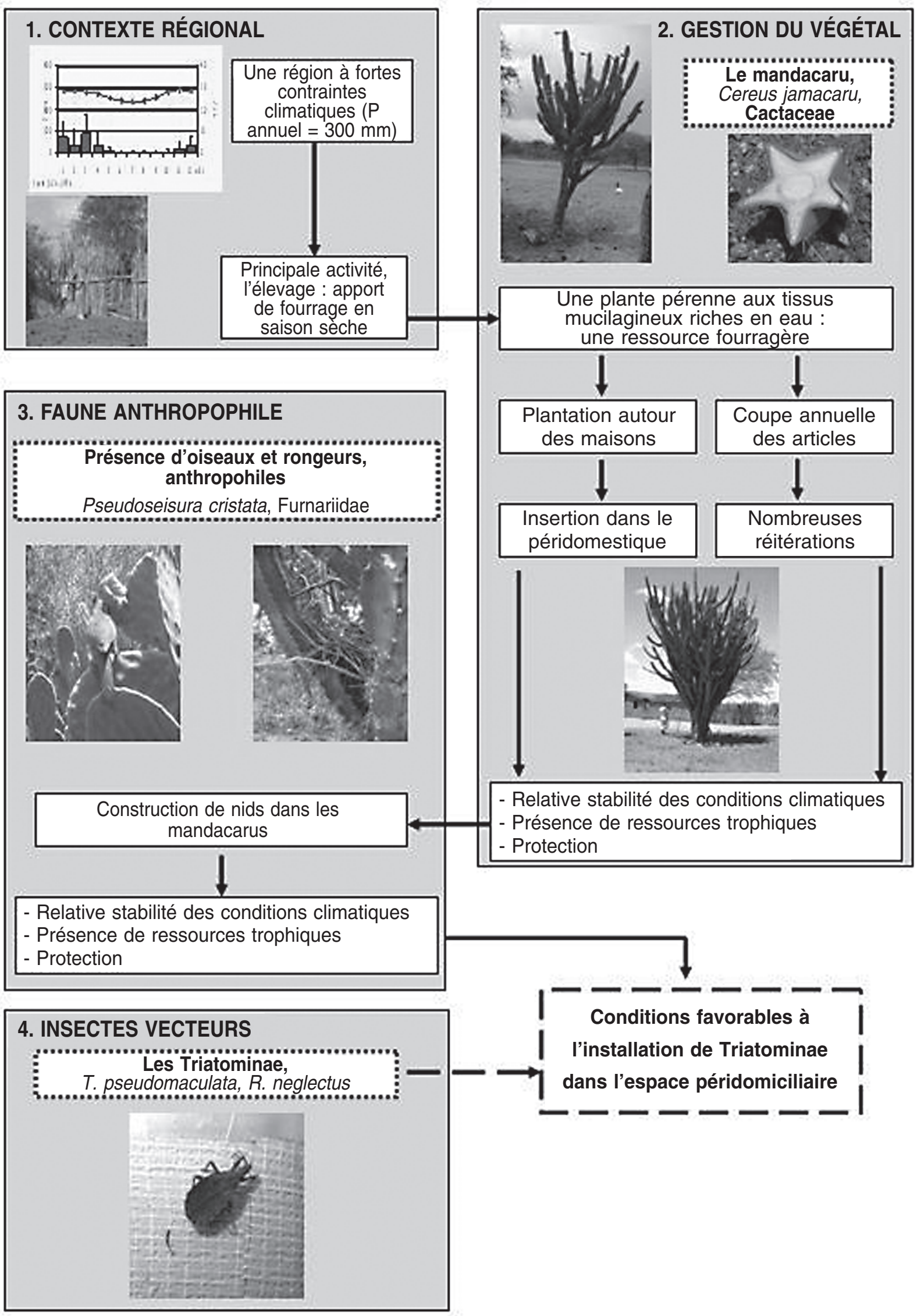

Fig. 4. - Relations entre gestion du "mandacaru" et présence de nids de Triatominae. 
apport de fourrage en saison sèche qui est fourni par les articles gorgés d'eau des "mandacarus" plantés aux alentours des habitations; 3) la coupe périodique des articles entraîne la formation de nombreuses réitérations qui donnent aux cactus un port touffu, formant ainsi un habitat relativement protégé pour les oiseaux ou les rongeurs; 4) l'insertion des "mandacarus" dans des palissades qui relient différents espaces consacrés à l'élevage facilite l'installation des Triatominae, principalement de T. pseudomaculata, qui y trouvent des ressources trophiques et des conditions climatiques plus stables que dans le milieu sylvestre.

Ce schéma s'applique tant au péridomicile en activité où les ressources trophiques (gens, bétail, petit élevage,...) sont abondantes, qu'au péridomicile abandonné où se maintiennent des "mandacarus" et autres espèces ligneuses, sources d'ombre et de fourrage pour le bétail qui divague. L'existence d'écotopes favorables aux Triatominae n'est donc pas strictement inféodée à la présence de l'homme; ces derniers se maintiennent indépendamment de la présence humaine et s'inscrivent dans le paysage, vu la longévité de ce cactus. Cette analyse et celle de Canale et al. (2000) dans le nord-ouest de l'Argentine soulignent l'importance des éléments végétaux comme habitats des Triatominae dans le péridomicile et la nécessité de les disséquer systématiquement.

Nos résultats mis en perspective avec les travaux de Walter et al. (2005) menés dans la même région permettent de supposer une nette structuration des habitats, et des ressources trophiques, des Triatominae autour des unités domiciliaires. Cependant, si les nids dans les "mandacarus" étudiés par nous constituent un écotope favorable à deux espèces de Triatominae, $R$. neglectus et T. pseudomaculata, Walter et al. (2005) soulignent que la présence de ces cactus dans un rayon de $30 \mathrm{~m}$ autour des habitations est corrélée à l'infestation des habitations par T. brasiliensis Neiva, 1911 mais non par T. pseudomaculata.

Du point de vue méthodologique, cet exemple souligne l'intérêt d'une démarche pluridisciplinaire pour la compréhension de la distribution des insectes vecteurs. Celle-ci doit prendre en compte les différentes formes d'action de l'homme sur le milieu, ce n'est pas tant la présence de l'espèce $C$. jamacaru dans le péridomicile que son mode de gestion qui est une variable indicatrice de la présence de Triatominae. Cette recherche souligne aussi la nécessité d'une approche régionale de l'écologie des vecteurs. Ainsi, nous avons mené la même étude dans le sud-est du Piauí, autre région semi-aride, mais aux précipitations doubles de celles de Curaçá. Le rôle du "mandacaru" comme plante fourragère devient secondaire et aucun nid n'a été observé dans les 37 cactus relevés.

À Curaçá, la question du rôle des "mandacarus" dans le péridomicile est cependant posée. Peuvent-ils être considérés comme formant une zone tampon où les insectes trouvent les conditions nécessaires à leur alimentation et à leur reproduction sans se déplacer audelà ou, au contraire, s'agit-il d'une zone d'attraction dans un processus de colonisation de l'habitat humain? Cette deuxième hypothèse ne peut être exclue car tant R. neglectus que T. pseudomaculata ont déjà été capturés colonisant les habitations dans plusieurs états du centre et du nord-est brésilien (Silveira \& Vinhaes, 1999).

\section{REMERCIEMENTS}

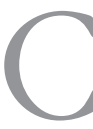

ette recherche a été réalisée et financée dans le cadre de la coopération IRD-UR 16/CNPq-Fiocruz n 910157/00-6 "Écologie du paysage, dynamique des agroécosystèmes et identification du risque : le cas de la trypanosomose américaine", coordination d'A-M Jansen (Fiocruz) et L. Emperaire (IRD) avec la participation du Centre de développement durable de l'Université de Brasília et de la Fondation nationale de santé du Ministère de la santé.

Nous remercions Agenor José Ferreira et Geraldo Sales de la Funasa, $15^{\text {ème }}$ DIRES-BA pour leur participation et appui lors de cette recherche, Rodrigo Gurgel et Guy Mejía, étudiants de master du Laboratoire de parasitologie de l'Université de Brasília, ainsi que le professeur Cesar Cuba-Cuba, responsable de ce laboratoire. Nos remerciements s'adressent aussi aux habitants de Macambira, de la Fazenda São Francisco et de Malhada da Pedra (commune de Curaçá-Bahia) qui ont accepté la réalisation de ces recherches.

\section{RÉFÉRENCES}

Alencar J.E. A Doença de Chagas no Estado do Ceará. Contribuição para o Conhecimento da sua Epidemiologia Regional. Fortaleza, Faculdade de Medicina, Universidade Federal do Ceará, 1980.

BRÉsIL, IBGE. Censo demográfico - Características da população e dos domicílios, Rio de Janeiro, IBGE, 2000, 519 p.

Canale D.M., Cecere M.C., Chuit R. \& Gurtler R.E. Peridomestic distribution of Triatoma garciabesi and Triatoma guasayana in north-west Argentina. Medical and Veterinary Entomology, 2000, 14, 383-390.

Carcavallo R.U. \& Martínez A. Biología, ecología y distribución geográfica de los triatominos americanos, in : Factores biológicos y ecológicos en la enfermedad de Chagas. Carcavallo R.U., Rabinovich J.E. \& Tonn R.J. (eds), OPSECO/MSAS-SNCH, Buenos Aires, 1985, vol. 1, 149-208.

Carcavallo R.U., Rodríguez M., Salvetella R., Curto S., Sherlock I., Galvão C., Rocha D., Galíndez I., Otero M.A., Arocha O., Martínez A., Da Rosa J., Canale D., Farr T. \& BARATA J. Habitats and related fauna, in: Atlas of Chagas disease vectors in the Americas. Carcavallo R.U., Galín- 
dez I., Jurberg J. \& Lent H. (eds), Fiocruz, Rio de Janeiro, 1998, vol. II., 561-600.

DCA-UFPB. Dados climatológicos do Nordeste do Brasil $<$ http://dca.ufpb.br>

LenT H. \& Wygodzinski P. Revision of the Triatominae (Hemiptera, Reduviidae), and their significance as vectors of Chagas' Disease. Bulletin of the American Museum of Natural History, 1979, 163, 127-520.

Lopes E. Caminhos de Curaçá, Curaçá, Gráfica Franciscana (éd. auteur), 2000, 260 p.

Marroquín R., Rosales R., Bor S., Guevara F. \& Monroy C. Hallazgo de Triatoma ryckmani (Hemiptera: Reduviidae) en 10,000 $\mathrm{m}^{2}$ del bosque espinoso y evaluación de parasitemia como vector de la enfermedad de Chagas, in: Memorias IV Congreso de la Sociedad Mesoamericana para la Biología y la Conservación, Ciudad de Panamá, Panamá, 2000a.

Marroquín R., Bor S., Guevara F. \& Monroy C. Adición a la descripción y primera descripción de los estadios ninfales I, II, III, IV, y V de Triatoma ryckmani (Hemiptera: Reduviidae), in: Memorias IV Congreso de la Sociedad Mesoamericana para la Biología y la Conservación, Ciudad de Panamá, Panamá, 2000b.

Sick H. Ornitologia brasileira, Brasília, UnB, Linha Gráfica Ed., 1984, 2 vol., 827 p.

Silveira A.C. \& VINHAEs M.C. Elimination of vector-borne transmission of Chagas disease. Memórias do Instituto Oswaldo Cruz, 1999, 94 (suppl. I), 405-411.

Vezzani D., Schweigmann N.J., Pietrokovsky S.M. \& WisniVESKY-COLLI C. Characterization of Triatoma guayasana biotopes in hardwood forest of Santiago del Estero, Argentina. Memórias do Instituto Oswaldo Cruz, 2000, 96 (4), 459-466.

Walter A., Pojo do Rego I., Ferreira A.G. \& Rogier C. Risk factors for reinvasion of human dwellings by sylvatic triatomines in northern Bahia State, Brazil. Cadernos de Saúde Pública, 2005, 21 (3), 974-978.

Reçu le $1^{\text {er }}$ août 2005 Accepté le 8 février 2006 